\title{
Bone-cement interface of the glenoid component: Stress analysis for varying cement thickness
}

\author{
Alexandre Terrier ${ }^{\mathrm{a}, *}$, Philippe Büchler ${ }^{\mathrm{a}}$, Alain Farron ${ }^{\mathrm{b}}$ \\ a Laboratoire de Recherche en Orthopédie, STI-IGBM-LRO, Bâtiment AA.B, Station 15, Ecole Polytechnique Fédérale de Lausanne (EPFL), \\ 1015 Lausanne, Switzerland \\ ${ }^{\text {b } H o ̂ p i t a l ~ O r t h o p e ́ d i q u e ~ d e ~ l a ~ S u i s s e ~ R o m a n d e, ~ U n i v e r s i t e ́ ~ d e ~ L a u s a n n e, ~ L a u s a n n e, ~ S w i t z e r l a n d ~}$
}

Received 16 December 2004; accepted 30 March 2005

\begin{abstract}
Background. Although shoulder arthroplasty is an accepted treatment for osteoarthritis, loosening of the glenoid component, which mainly occurs at the bone-cement interface, remains a major concern. Presently, the mechanical effect of the cement mantel thickness on the bone-cement interface is still unclear.

Methods. Finite element analysis of a prosthetic scapula was used to evaluate the effect of cement thickness on stresses and micromotions at the bone-cement interface. The glenoid component was all-polyethylene, keeled and flat back. Cement mantel thickness was gradually increased from 0.5 to $2.0 \mathrm{~mm}$. Two glenohumeral contact forces were applied: concentric and eccentric. Two extreme cases were considered for the bone-cement interface: bonded and debonded.

Findings. Within cement, stress increased as cement thickness decreased, reaching the fatigue limit below $1.0 \mathrm{~mm}$. Bone stress was below its ultimate strength and was minimum between 1.0 and $1.5 \mathrm{~mm}$. Interface stress was close to the interface strength, and also minimum between 1.0 and $1.5 \mathrm{~mm}$. Both the decentring of the load and the debonding of the interface increased the stress.

Interpretation. A cement thinning weakens the cement, but also the bone-cement interface, along the back-keel edges. Conversely, a cement thickening rigidifies the cemented implant, consequently increasing interfacial stresses and micromotions. To avoid both excessive cement fatigue and interface failure, an ideal cement thickness has been identified between 1.0 and $1.5 \mathrm{~mm}$.
\end{abstract}

(c) 2005 Elsevier Ltd. All rights reserved.

Keywords: Shoulder arthroplasty; Loosening; Cement; Interface

\section{Introduction}

Unconstrained total shoulder arthroplasty has become an accepted and common treatment for osteoarthritis of the shoulder. However, despite the success of the procedure in terms of pain relief and gain in functionality $(85-95 \%)$, a major concern remains regarding the fixation and longevity of the glenoid component, which is generally all-polyethylene and cemented (Boi-

\footnotetext{
* Corresponding author.

E-mail address: alexandre.terrier@epfl.ch (A. Terrier).
}

leau et al., 2002). The clinical loosening of the glenoid component is the primary cause of failure; it has been correlated to fibrous tissue formation (Torchia et al., 1997), which is associated to radiolucent lines at the bone-cement interface, observed in $30-95 \%$ of patients at follow-up (Wirth and Rockwood, 1996).

The fibrous tissue formation and the loosening are caused by multiple factors; the most important is certainly the level of micromotion of the implant relative to the underlying bone (Aspenberg and Herbertsson, 1996). Another important factor is the presence of debris of cement, which may combine to the micromotion, to induce an inflammatory response. Both are linked 
to the level of the stress near the bone-cement interface: above a critic level, stress may induce an interface failure (micromotion) and/or a fatigue failure of the cement (debris). One of the main mechanical causes of the loosening is therefore the repetitive loading of the implant over some critical level. Finally, the stress state at bonded interface may be used to evaluate the primary stability of the implant, but also give some insight of the long-term stability.

It has already been demonstrated that the cementing technique may improve the long-term survival of the cemented glenoid component (Norris and Lachiewicz, 1996). Moreover, in vitro tests have revealed that the thickness of the cement mantle influences the primary stability of cemented glenoid components (Nyffeler et al., 2003). Numerical models were also used to analyse the effect of cement thickness around glenoid implants: a 2D model showed that a 50\% increase of cement thickness increased the stress within the trabecular bone adjacent to the cement up to 50\% (Stone et al., 1999); in a 3D model, a comparison between normal and double cement thickness revealed a $10 \%$ increase of bone stress, and a $30 \%$ increase of cement stress (Couteau et al., 2001). These numerical studies, which were not focused on cement thickness effect, only considered fully bonded interfaces, and did not provide relative micromotions at the bone-cement interface.

Despite the above studies, there is no clear recommendation for the thickness of the cement mantel. Thus, there is still a lack of information concerning the biomechanical mechanisms relating cement thickness on the stress transfer from the glenoid surface to the bonecement interface. Since these mechanisms are essential for the primary and long-term stability of the glenoid component, a three-dimensional finite element model of a prosthetic scapula was developed to analyse this parameter. A cemented all-polyethylene glenoid component was inserted, and increasing values of the cement thickness were considered. The glenoid implant stability was evaluated from several mechanical quantities near bone-cement interface. Two extreme cases were analyzed: fully bonded and fully debonded, which corresponds respectively to the primary implant stability immediately after surgery, and to a fully loose implant. The first case was used to estimate the occurrence of the (progressive) loosening process, while the second case was used to give some insight of the stress state near the interface when it has failed.

\section{Methods}

The three-dimensional geometry of the scapula was reconstructed from $1 \mathrm{~mm}$ computed tomography (CT) slices of an intact cadaver shoulder, without any macroscopic or radiological signs of pathology. The glenoid implant was all-polyethylene, keeled and flat backed; its articular surface was spherical with a radius of curvature of $30 \mathrm{~mm}$. It was positioned into the reconstructed scapula in such a way that the glenoid surface fit the cartilage surface of the scapula (centre point and orientation), and according to surgeon recommendations. A cement mantel of uniform thickness was added around the implant. At the bony side, the cement mantel edges were smoothed with a $2 \mathrm{~mm}$ radius fillet to avoid sharp contacts at the bone-cement interface. The bone resection of the glenoid corresponded to the exact volume occupied by the cemented implant. This virtual arthroplasty procedure was repeated with four different cement thicknesses: 0.5, 1.0, 1.5 and $2.0 \mathrm{~mm}$ (Fig. 1).

Bone was considered as a linear elastic material, with non-homogeneous properties related to its density (Rice et al., 1988; Schaffler and Burr, 1988), which was derived from the CT data. Implant and cement were homogeneous linear elastic bodies (implant: $E=500 \mathrm{MPa}$, $v=0.4$; cement: $E=2000 \mathrm{MPa}, v=0.3$ ). The cement implant interface was fully bonded, whereas at the bone-cement interface two cases were considered: fully bonded and fully debonded. In the latter case perpendicular and tangential micromotions were allowed at this

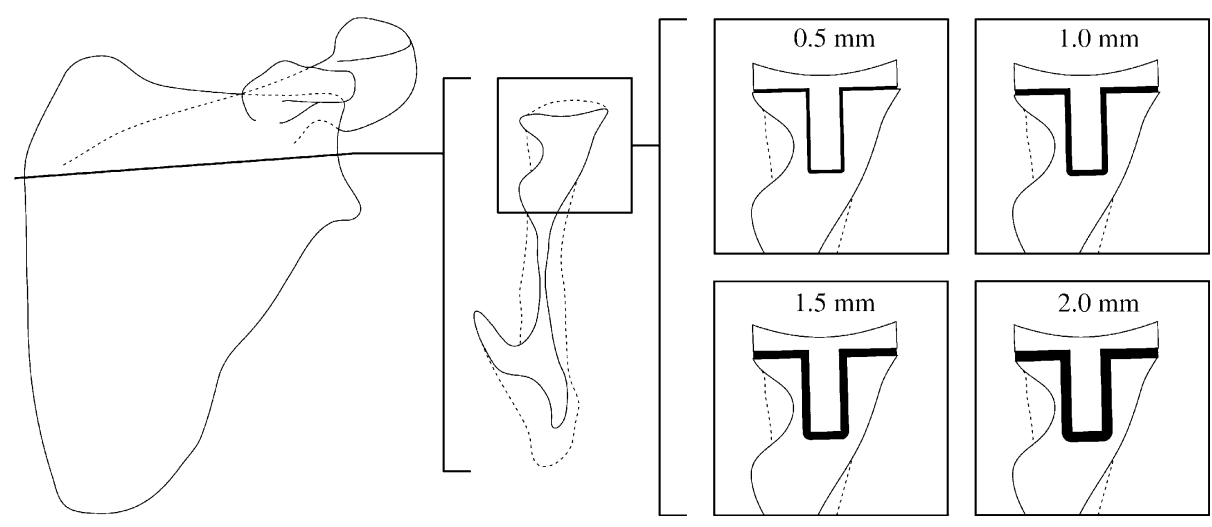

Fig. 1. Cut view of the scapula (in the plane of the glenoid) showing the glenoid component implanted with four different thicknesses of the cement mantel. 
interface, and a friction coefficient of 0.6 was considered (Walker, 1977; Ramaniraka et al., 2000).

The scapula was rigidly fixed at the insertion points of the trapezius and rhomboideus muscles and at some points of its anterior side, which corresponded to the contact with the thorax (Buchler et al., 2002). On the glenoid surface, two force amplitudes were considered: $400 \mathrm{~N}$, which corresponded to the maximal glenohumeral force during abduction (van der Helm, 1994), and an arbitrary moderate force of $100 \mathrm{~N}$. In addition, two force locations were considered: concentric and eccentric (Fig. 2). The distribution of the force over the surface was derived from the Hertz theory for nonconforming surfaces in contact (humeral implant radius: $26 \mathrm{~mm})$.

The scapula was discretized with second order tetrahedral elements, and the cement and implant with linear hexahedral elements (Fig. 2). The size of the elements was maintained below $1 \mathrm{~mm}$ near the interface, which generated over 60,000 elements for the scapula, 7000 for the cement and 6000 for the implant, resulting in over 500,000 degrees of freedom. Static non-linear stress analyses were performed with Abaqus 6.3 (Hibbitt, Karlsson and Sorensen, Inc.), allowing for largedisplacement effects and contact nonlinearities. The bone-cement interface was either restrained or allowed to debond (and slip). Several mechanical quantities were calculated near the bone-cement interface: the maximum (tensile) and minimum (compressive) principal stress within the cement mantel, the Von Mises stress within the underlying bone (adjacent to the interface), the pressure (and tension), shear stress, perpendicular

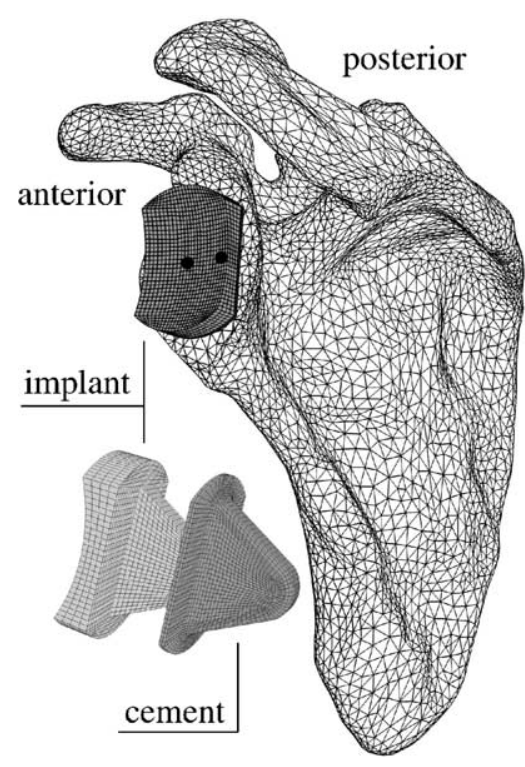

Fig. 2. Finite element mesh of the prosthetic scapula with a detail view of the glenoid component and cement mantel. Black points on the glenoid surface represent the centre of the concentric and eccentric load distributions. (debonding) and tangential (slipping) micromotions at the bone-cement interface. Peak values and spatial distributions were considered.

\section{Results}

The results were obtained for the maximal and moderate force, in the four different cases: concentric force and bonded interface, eccentric force and bonded interface, concentric force and debonded interface, and eccentric force and debonded interface. They are presented successively for the cement mantel, the underlying bone, and the bone-cement interface, only for the maximal force. For sake of simplicity, only peak values of stresses and micromotions are represented graphically (Figs. 4-7). To verify that the stress concentration was smoothly and continuously distributed over several elements rather than on a single node (numerical singularity), the contact pressure distribution at the bonecement interface is displayed (Fig. 3).

\subsection{Cement mantel}

In all four cases, there was a stress increase as cement thickness decreased from 2.0 to $0.5 \mathrm{~mm}$ (Fig. 4). The eccentric loading induced an increase of both tensile $(+20 \mathrm{MPa})$ and compressive (+15 MPa) stress. The debonding of the bone-cement interface had almost no effect. In the concentric case, the stress distribution was symmetric (about the frontal plane), and stress

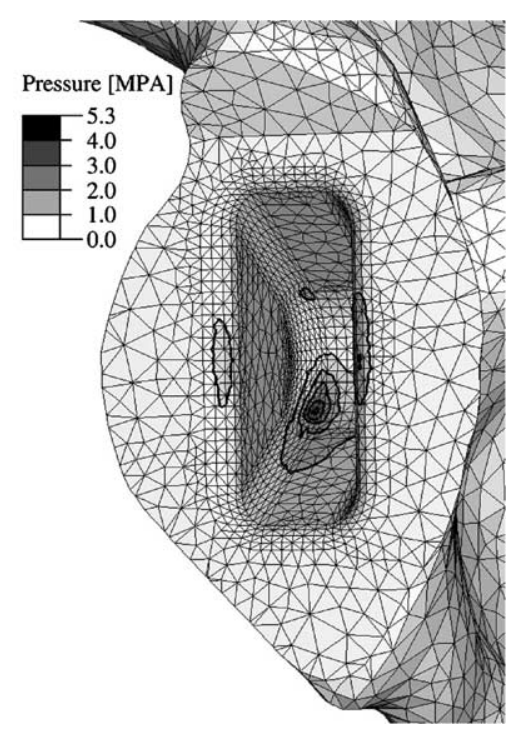

Fig. 3. Distribution of the contact pressure at the bone-cement interface, for the concentric force and bonded case, with $2 \mathrm{~mm}$ of cement thickness. This detail view confirms that, although the stress concentration was restricted to a small region (anterior inferior corner of the keel), it was smoothly and continuously distributed over several elements of the mesh. 

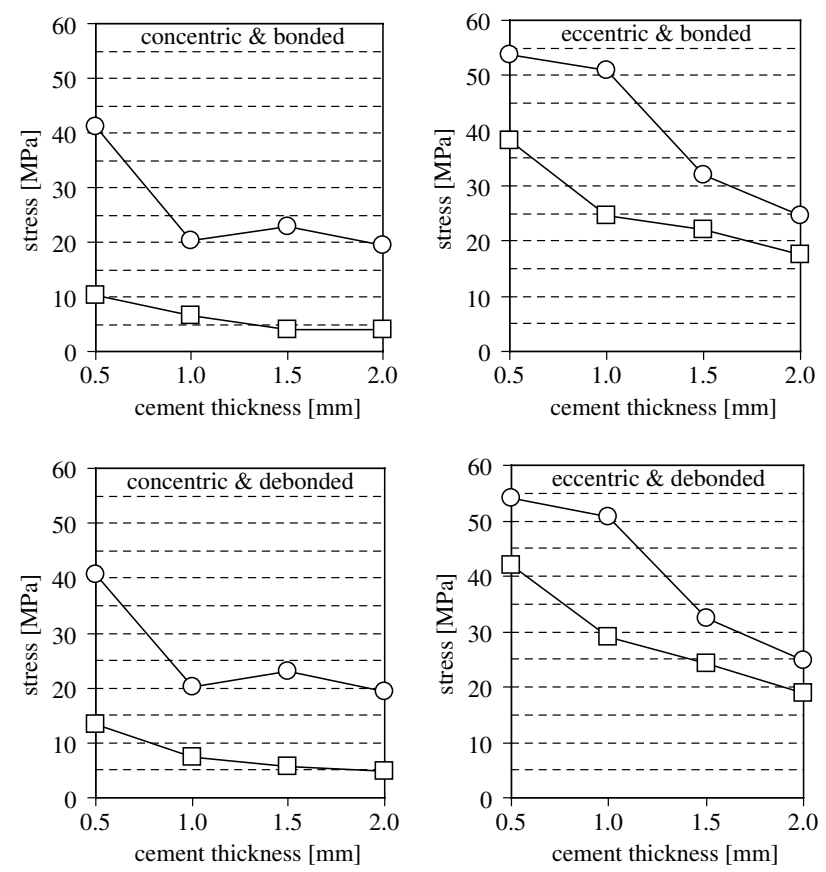

Fig. 4. Peak values of maximum principal stress (square) and the minimum principal stress (circle) within cement.

concentration was mainly located along the back-keel edges; in the eccentric case, stress concentration was located at the posterior side of the back. In all cases, peak stress was located in a small volume fraction (3\% volume above $5 \mathrm{MPa}$ ).

\subsection{Underlying bone}

There was a minimum of Von Mises stress between 1.0 and $1.5 \mathrm{~mm}$ of cement thickness in all cases, except for the eccentric/bonded case, for which stress was almost constant (Fig. 5). Stress increased drastically with eccentric loading $(+25 \mathrm{MPa})$, and only slightly with interface debonding. In the concentric case, the stress concentration was mainly located at the keel tip, but also along the back-keel edges as thickness decreased; it was located at the posterior side of the back in the eccentric case.

\subsection{Bone-cement interface}

There was a global increase of the interfacial stresses as thickness decreased from 1.0 to $0.5 \mathrm{~mm}$ (Fig. 6). From 1.0 to 2.0, there was an increasing trend. In the bonded case, shear stress was only slightly modified by cement thickness. The eccentric loading increased pressure, tension and shear stress. Debonding induced a significant increase of pressure $(+12 \mathrm{MPa})$ and a slight decrease of shear stress $(-1 \mathrm{MPa})$ in the concentric case, but increased both pressure $(+6 \mathrm{MPa})$ and shear $(+6 \mathrm{MPa})$ in the eccentric case. In the concentric case,
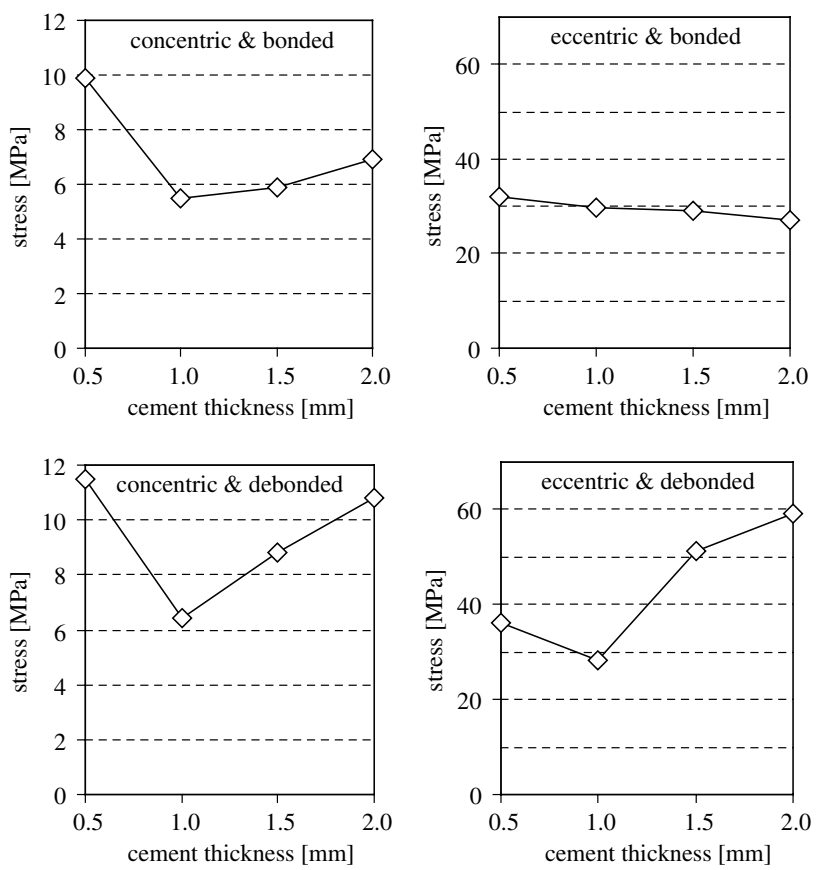

Fig. 5. Peak values of Von Mises stress at the underlying bone surface.
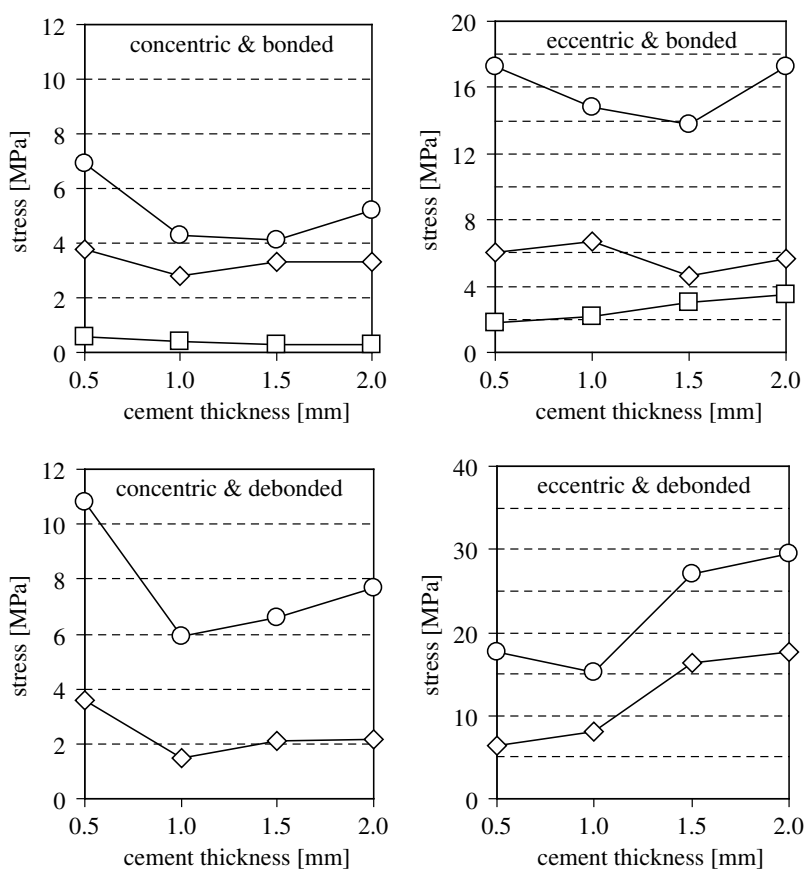

Fig. 6. Peak values of pressure (circle), tension (square) and shear (diamond) stress at the bone-cement interface.

the stress concentration was mainly located at the keel tip, but also at the back-keel edges as thickness decreased; it was located at the posterior side of the back in the eccentric case. Micromotions were evidently only nonzero in the debonded case. In the concentric case, they were moderate and almost constant, whereas in the eccentric case, they were very important and 

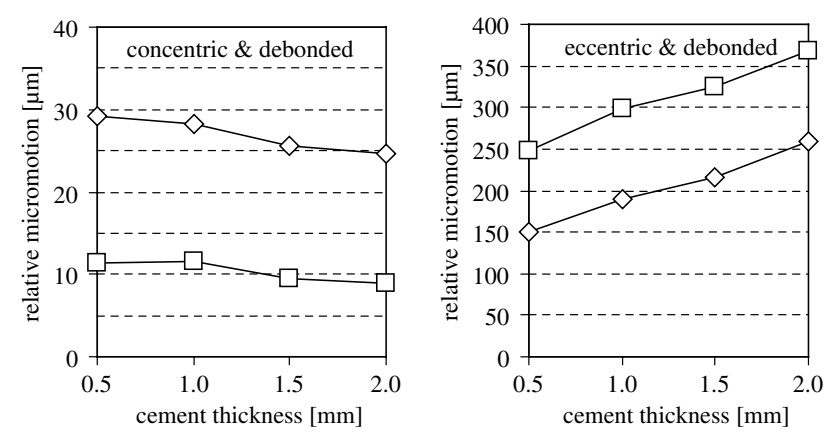

Fig. 7. Peak values of perpendicular (square) and tangential (diamond) micromotions at the bone-cement interface.

increased regularly (Fig. 7). In the concentric case, perpendicular micromotions (debonding) were clearly located at the anterior and posterior side of the keel, while tangential micromotions (slipping) were located more or less around the keel. In the eccentric case, micromotions were located at the anterior side of the back.

\subsection{Summary of the results}

Within cement, increase of cement thickness induced a continuous decrease of stress. Within bone, and at the bone-cement interface, there was a stress decrease from 0.5 to 1.0 and a increase from 1.0 to 2.0 , suggesting a minimum between 1.0 and $1.5 \mathrm{~mm}$ of cement thickness. The eccentric loading, as well as the debonding of the interface, induced an overall increase of stress. Tangential micromotions remained moderate and almost constant in the concentric case, but were excessive and increasing in the eccentric case. Peak stress was at the keel tip, but also along the back-keel edges as cement thickness decreased. Micromotions were at the keel faces in the concentric case, but under the back in the eccentric case. In the case of the moderate force, which was not presented above, all values were more or less four times lower.

\section{Discussion}

The objective of this study was to evaluate the influence of the cement thickness on the primary and longterm stability of the glenoid component after total shoulder arthroplasty. By means of a finite element model of a scapula including a prosthetic glenoid, the stresses within cement, underlying bone and at the bone-cement interface were analysed, for a perfectly bonded bone-cement interface and for a completely loose one. A concentric and eccentric (posterior) loading were simulated. The influence of the cement thickness on the glenoid stability has been confirmed, and an optimal value between 1.0 and $1.5 \mathrm{~mm}$ has been identified.
Two different quantities were used to estimate the failure (facture) of bone and cement. Bone is widely regarded as a ductile material, particularly in compression (Yeh and Keaveny, 2001), while cement is clearly a brittle material. For ductile materials, Von Mises stress is the criterion traditionally used to predict failure, which occurs when Von Mises stress equals the ultimate strength. For brittle materials, failure takes places when the maximal or minimal principal stress exceeds the ultimate strength in tension or compression respectively. At the bone-cement interface, the stress tensor was naturally decomposed into its perpendicular and tangential contribution relatively to the boundary surface. In addition, the perpendicular contribution was separated into the inward (pressure) or outward (tension) direction, since cement is stronger in compression $(\sim 100 \mathrm{MPa})$ than in tension $(\sim 30-50 \mathrm{MPa})$.

A special attention was paid to avoid numerical artefacts caused by sharp corners or a coarse mesh. All edges of the bone-cement interface were rounded and at least six elements were used along this $90^{\circ}$ arc circle, avoiding therefore angles greater than $15^{\circ}$ (Fig. 3). Linear hexahedral element would have been well suited for the whole model; unfortunately, it was too difficult to accurately fill in the complex geometry of the scapula with this element shape. This problem was solved by using tetrahedral elements, for which automatic algorithms can fill in any geometry. As linear tetrahedral elements are not usually as good as linear hexahedral element, particularly when contact is involved, second order tetrahedral elements were used, as recommended by Abaqus for contact problems. Finally, hexahedral elements were used for the cement and implant, and second order tetrahedral elements for the scapula. The mesh refinement was based on simple rules rather than a convergence study. For the cement mantel, at least two layers of elements were used. The global meshing refinement of the cement was then constrained in such a way to avoid, as much as possible, excessive distortion of the ideal cubic shape of hexahedral elements (Fig. 2). The meshing of the implant was continuous with the one of the cement, sharing the same node location at the adjacent surfaces, and resulting in a similar element size $(\sim 0.25-1.0 \mathrm{~mm})$. The same rule was applied to the scapula: a similar nodes density (and element size) was used, at least at the bone-cement interface, although it was not possible to require that all nodes of the bone resection surface share the same location than the nodes of the cement, since bone was filled in with tetrahedral elements and cement with hexahedral elements.

The predicted values for cement stress were within the same range of other similar FE studies: $6 \mathrm{MPa}$ (Lacroix et al., 2000), 1.3-1.4 MPa (Couteau et al., 2001), 4 $6 \mathrm{MPa}$ (Murphy et al., 2001), 1-10 MPa (Gupta et al., 2004). However, the observed cement stress decrease as cement thickness increase was contrary to the only 
study that discussed this effect (Couteau et al., 2001), by doubling the cement thickness from $2-4$ to $4-8 \mathrm{~mm}$, which is quite high. This huge cement thickness may have required a resected surface that was much closer to hard cortical bone, also requiring a larger removal of the subcondral bone. Therefore, glenohumeral force was transmitted more directly to the cement, which was much more in contact with harder bone, inducing a harder contact, and thus a higher stress within the cement. This effect may have been accentuated by the mechanical properties used for cortical bone, which had a uniform and quite high stiffness $(16,000 \mathrm{MPa})$ compared to usually accepted values $(8000 \mathrm{MPa})$. This hypothesis may be confirmed by the relative decrease $(10 \%)$ of the overall displacement of the cement that they obtained for the thicker cement layer. Finally, the relationship between cement stress and cement thickness observed in the present study was also reported for femoral cemented stems (Lee et al., 1993; Estok et al., 1997; Ramaniraka et al., 2000; Schmolz et al., 2000; Ayers and Mann, 2003).

Cement compressive and tensile stress were far below ultimate strength (Lewis, 1997). However, since loosening has been related to cement debris (Topoleski et al., 1990), resulting from cement micro-cracks, one may consider here a fatigue failure criterion. Following the developments of Krause et al. (1988), Murphy and Prendergast (2000) obtained a relationship between maximum principal stress and probability of survival to failure for hand-mixed cement, for 10 million cycles. According to their theory, a stress higher than $4 \mathrm{MPa}$ would result in a probability of failure lower than $75 \%$. In the same way, Davies et al. (1988) reported a Weibull fatigue life of about 245,000 cycles at $7 \mathrm{MPa}$ and 1600 at $15 \mathrm{MPa}$, for uncentrifugated cement and trabecular bone. According to these experimental studies, $5-7 \mathrm{MPa}$ is a generally accepted value for microcracks initiation within cement (Lacroix et al., 2000). In the concentric case, tensile stress exceeded this value when cement thickness was below $1 \mathrm{~mm}$; in the eccentric case, tensile stress was always above it.

Within bone, Von Mises stress was also far below the bone ultimate strength, which was calculated locally from bone density (Rice et al., 1988). Even in the most unfavourable case (eccentric/debonded $/ 2 \mathrm{~mm}$ ), bone stress remained below the ultimate strength, as observed by Lacroix et al. (2000). Contrary to the cement, there was an increase of the stress as cement thickness increased above $1.0 \mathrm{~mm}$, which confirms previous 2D (Stone et al., 1999) and 3D (Couteau et al., 2001) models.

Interfacial strength measurements at the bonecement interface are reported between 2 and $40 \mathrm{MPa}$ (Mann et al., 1997). Mechanical tests of interfacial tensile strength between cement and trabecular bone under pure tension were reported in the order of $7.5 \mathrm{MPa}$ (Kusleika and Stupp, 1983). Another experimental study reported a shear strength between 1 and $6 \mathrm{MPa}$, according to the amount of the interdigitated bone and bone density (Mann et al., 1997, 1999). More specifically, pull-out strength measurements of glenoid component pegs (Nyffeler et al., 2003) provides a rough estimate of $3 \mathrm{MPa}$ for the shear strength of the bone-cement interface. Based on a Hoffman criterion, Gupta et al. (2004) used the Hoffman number (Huiskes and van Rietbergen, 1995) to analyse the failure of the fully bonded bone-cement interface of a keeled glenoid implant. They derived a shear strength between 3.8 and $6.9 \mathrm{MPa}$, a tensile strength between 2.4 and $4.4 \mathrm{MPa}$, and a compressive strength between 4.6 and $8.9 \mathrm{MPa}$. According to the interface failure strengths reported above, the interfacial peak stress obtained here were close to these limit values, and we confirm that the bone-cement interface was likely to fail (Couteau et al., 2001; Gupta et al., 2004), at the tip of the keel, as noted by Gupta et al., but also along the back-keel edges when cement thickness was below $1.5 \mathrm{~mm}$. The effect of cement thickness on interfacial stress was moderate, although there was a minimum of interfacial stress between 1.0 and $1.5 \mathrm{~mm}$ of cement thickness.

In the extreme situation of full debonding (implant loosening), tangential micromotions remained close to the limit $(20 \mu \mathrm{m})$ for bone ingrowths and implant stability (Jasty et al., 1997) in the concentric case. This value was largely exceeded in the eccentric case, promoting fibrous tissue formation and the implant loosening process. Moreover, when the implant starts to debond, cement particles, resulting from cement micro-fractures, may migrate towards regions of high slipping and accentuate the effect of the micromotions on fibrous tissue formation (Goodman, 1994; Aspenberg and Herbertsson, 1996). In the case of the eccentric load, the debonding was located at the anterior plate, and can be clearly interpreted as a sign of the well-known rocking-horse effect.

In summary, this study has demonstrated that a cement thinning weakens the cement mantel by increasing its stress up to fatigue failure. Moreover, below $1.0 \mathrm{~mm}$, excessive peak stress appeared at the bone-cement interface, around the back-keel edges. Conversely, a thickening of the cement mantel rigidifies the cemented implant, consequently increasing the stress at the bone-cement interface, but also within the underlying bone. To avoid both excessive cement fatigue and failure of the bonecement interface, an ideal value of $1 \mathrm{~mm}$ was identified. In the case of the moderate force, the mechanical behaviour was similar, but the limit values were not exceeded.

This numerical study presents some limitations. A healthy shoulder was used for this study instead of an osteoarthritic one. However, a different bone density would mainly change bone and bone-cement interface strength, but not the tendency observed here. Results were obtained for a specific (keel and flat) glenoid 
implant design; we may however assume that the same mechanisms would occur for peg or convex implants.

The cement, which was idealized by a layer of uniform thickness with rounded edges at the bony side, may have a much more complex geometry. The microstructure of the bone-cement interface may however be represented by the macroscopic interfacial strength, which depends on the penetration depth of the cement. Actually, the cement may spread into the trabecular bone and form a large cement block around the keel, especially with osteopenic glenoids. This was reported by an in vitro study (Nyffeler et al., accepted for publication), which used high resolution micro-CT to analyze the cementation around pegged glenoid components. This study showed that the cement layer between the back of the implant and the subcondral bone was between 0 and $2 \mathrm{~mm}$, and not always complete. Around the pegs, the cement mantel was complete, and in most cases cylindrical with a wall thickness between 0.3 and $1.3 \mathrm{~mm}$, except in one osteopenic specimen where the cement penetrated into the cancellous bone and formed a big cement block filling out the whole inferior half of the glenoid.

Only two extreme cases of interface adhesion were considered: fully bonded and fully debonded. In reality, loosening is a continuous process involving a continuous decrease of the failure strength of the interface, caused by fibrous tissue formation and bone resorption; these biological processes were not included here. However, even if the implant does not eventually fully debond, one may assume that the fully debonded case gives a rough estimate of the location and peak stress and micromotion. This method is of course a simplification of the continuous debonding process, which would require to be modelled by an evolution law, and not only by the initial and final state of the evolution. In the loose case, the friction coefficient corresponded to cortical bone and may be higher for trabecular bone; however, it may be consistent when compaction of trabecular bone is performed before implantation.

Only two loading cases were considered here. However, they are relevant for the activities of daily living. The force amplitude corresponded to an unloaded arm at 90 degrees of abduction (van der Helm, 1994). The concentric case should be related to the abduction in the scapula plane with an ideal glenoid component alignment, while the eccentric case could be related to anteflexion (van der Helm, 1994), but also to a glenoid misalignment. It was indeed demonstrated that retroversion of the glenoid component, which is frequently reported (Walch et al., 1999), shifts the contact point towards the posterior rim of the implant (Nyffeler et al., 2000; Farron et al., 2004).

The present study is based on a finite element model that was not validated against specific experimental measurements. However, the material properties for bone and some of these numerical results can be compared to other experimental studies. The apparent density of cancellous bone in the glenoid, which was derived from $\mathrm{CT}$, was found between 0.3 and $0.6 \mathrm{~g} / \mathrm{cm}^{3}$. According to the relation that we used, it corresponded to an elastic modulus between 170 and $500 \mathrm{MPa}$. For cortical bone, the same method gave an elastic modulus between 4000 and $8000 \mathrm{MPa}$. These values are in agreement with experimental measurements (Frich et al., 1997; Mansat et al., 1998; Anglin et al., 1999), and are also consistent with other models (Orr et al., 1988; Stone et al., 1999; Lacroix et al., 2000; Murphy et al., 2001). Using similar mechanical properties for bone, cement and polyethylene, the principal stress within cement were in the same range than other 3D FE models (Lacroix et al., 2000; Murphy et al., 2001; Gupta et al., 2004). In a cadaver study, strain measurements were performed at four locations of the scapula, implanted with a similar glenoid component, and under a concentric $700 \mathrm{~N}$ load (Barea, 1998). Since strain measurement were quite different for each tested scapula, a strict comparison with our model was impossible. However, the maximal measured strain $\left(680 \times 10^{-3}\right)$ was on the same order than the strain $\left(300-1500 \times 10^{-3}\right)$ calculated approximately at the same locations and directions. In another cadaver study, the medio-lateral strain at the keel faces of a similar glenoid implant were measured, with very close loading conditions: a $400 \mathrm{~N}$ concentric force and a prescribed anterior-posterior translation (Karduna et al., 1998). For $4 \mathrm{~mm}$ of radial mismatch (which corresponds to our model), $2.2 \times 10^{-3}$ was measured in compression and $1.3 \times 10^{-3}$ in tension. These experimental values are relatively close to the values obtained by our model, in the same location and direction: $1.5 \times 10^{-3}$ in compression and $1.0 \times 10^{-3}$ in tension.

The clinical relevance of this study is clear: cement thickness is a key factor in glenoid component loosening, and consequently to the survival rate of total shoulder arthroplasty. Furthermore, results suggest an optimum cement thickness between 1.0 and $1.5 \mathrm{~mm}$. Practically, in order to achieve this constraint as much as possible, we would recommend to fill in any glenoid bone defects (at the bone resection surface) with bone graft, and/or perform a bone compaction before the cement insertion, to prevent cement to form large blocks around the implant. Moreover, future developments of new glenoid implants and associated instrumentation should be designed on the basis of a minimum $1.0 \mathrm{~mm}$ cement thickness, even if it may not be fully controlled.

\section{References}

Anglin, C., Tolhurst, P., Wyss, U.P., Pichora, D.R., 1999. Glenoid cancellous bone strength and modulus. J. Biomech. 32, 1091-1097. Aspenberg, P., Herbertsson, P., 1996. Periprosthetic bone resorption. Particles versus movement. J. Bone Joint Surg. Br. 78, 641-646. 
Ayers, D., Mann, K., 2003. The importance of proximal cement filling of the calcar region: a biomechanical justification. J. Arthroplasty $18,103-109$.

Barea, C., 1998. Modélisation 3D par la méthode des éléments finis d'une articulation scapulo-humérale. Application à l'étude des contraintes sur une épaule saine et avec différents types de glènes prothétiques. Thesis, Université Paul Sabatier Toulouse III.

Boileau, P., Avidor, C., Krishnan, S.G., Walch, G., Kempf, J.F., Mole, D., 2002. Cemented polyethylene versus uncemented metalbacked glenoid components in total shoulder arthroplasty: a prospective, double-blind, randomized study. J. Shoulder Elbow Surg. 11, 351-359.

Buchler, P., Ramaniraka, N.A., Rakotomanana, L.R., Iannotti, J.P., Farron, A., 2002. A finite element model of the shoulder: application to the comparison of normal and osteoarthritic joints. Clin. Biomech. 17, 630-639.

Couteau, B., Mansat, P., Estivalezes, E., Darmana, R., Mansat, M., Egan, J., 2001. Finite element analysis of the mechanical behavior of a scapula implanted with a glenoid prosthesis. Clin. Biomech. $16,566-575$.

Davies, J.P., O'Connor, D.O., Burke, D.W., Jasty, M., Harris, W.H., 1988. The effect of centrifugation on the fatigue life of bone cement in the presence of surface irregularities. Clin. Orthop., 156-161.

Estok II, D.M., Orr, T.E., Harris, W.H., 1997. Factors affecting cement strains near the tip of a cemented femoral component. J. Arthroplasty 12, 40-48.

Farron, A., Terrier, A., Büchler, P., 2004. Loosening of the prosthetic glenoid: influence of the subscapularis stiffness and the retroversion of the glenoid. 9th Congress on Surgery of the Shoulder, Washington, DC.

Frich, L.H., Jensen, N.C., Odgaard, A., Pedersen, C.M., Sojbjerg, J.O., Dalstra, M., 1997. Bone strength and material properties of the glenoid. J. Shoulder Elbow Surg. 6, 97-104.

Goodman, S.B., 1994. The effects of micromotion and particulate materials on tissue differentiation. Bone chamber studies in rabbits. Acta Orthop. Scand. Suppl. 258, 1-43.

Gupta, S., van der Helm, F.C., van Keulen, F., 2004. Stress analysis of cemented glenoid prostheses in total shoulder arthroplasty. J. Biomech. 37, 1777-1786.

Huiskes, R., van Rietbergen, B., 1995. Preclinical testing of total hip stems. The effects of coating placement. Clin. Orthop., 64-76.

Jasty, M., Bragdon, C., Burke, D., O'Connor, D., Lowenstein, J., Harris, W.H., 1997. In vivo skeletal responses to porous-surfaced implants subjected to small induced motions. J. Bone Joint Surg. Am. 79, 707-714.

Karduna, A.R., Williams, G.R., Iannotti, J.P., Williams, J.L., 1998. Total shoulder arthroplasty biomechanics: a study of the forces and strains at the glenoid component. J. Biomech. Eng. 120, 92-99.

Krause, W., Mathis, R.S., Grimes, L.W., 1988. Fatigue properties of acrylic bone cement: S-N, P-N, and $\mathrm{P}-\mathrm{S}-\mathrm{N}$ data. J. Biomed. Mater. Res. 22, 221-244.

Kusleika, R., Stupp, S.I., 1983. Mechanical strength of poly (methyl methacrylate) cement-human bone interfaces. J. Biomed. Mater. Res. 17, 441-458.

Lacroix, D., Murphy, L.A., Prendergast, P.J., 2000. Three-dimensional finite element analysis of glenoid replacement prostheses: a comparison of keeled and pegged anchorage systems. J. Biomech. Eng. 122, 430-436.

Lee, I.Y., Skinner, H.B., Keyak, J.H., 1993. Effects of variation of cement thickness on bone and cement stress at the tip of a femoral implant. Iowa Orthop. J. 13, 155-159.

Lewis, G., 1997. Properties of acrylic bone cement: state of the art review. J. Biomed. Mater. Res. 38, 155-182.

Mann, K.A., Ayers, D.C., Werner, F.W., Nicoletta, R.J., Fortino, M.D., 1997. Tensile strength of the cement-bone interface depends on the amount of bone interdigitated with PMMA cement. J. Biomech. 30, 339-346.

Mann, K.A., Werner, F.W., Ayers, D.C., 1999. Mechanical strength of the cement-bone interface is greater in shear than in tension. J. Biomech. 32, 1251-1254.

Mansat, P., Barea, C., Hobatho, M.C., Darmana, R., Mansat, M., 1998. Anatomic variation of the mechanical properties of the glenoid. J. Shoulder Elbow Surg. 7, 109-115.

Murphy, B.P., Prendergast, P.J., 2000. On the magnitude and variability of the fatigue strength of acrylic bone cement. Int. J. Fatigue 22, 855-864.

Murphy, L.A., Prendergast, P.J., Resch, H., 2001. Structural analysis of an offset-keel design glenoid component compared with a centerkeel design. J. Shoulder Elbow Surg. 10, 568-579.

Norris, B.L., Lachiewicz, P.F., 1996. Modern cement technique and the survivorship of total shoulder arthroplasty. Clin. Orthop., 7685.

Nyffeler, R.W., Anglin, C., Sheikh, R., Gerber, C., 2003. Influence of peg design and cement mantle thickness on pull-out strength of glenoid component pegs. J. Bone Joint Surg. Br. 85, 748-752.

Nyffeler, R.W., Meyer, D., Sheikh, R., Koller, B., Gerber, C., accepted for publication. The effect of cementing technique on structural fixation of pegged glenoid components in total shoulder arthroplasty. J. Shoulder Elbow Surg.

Nyffeler, R.W., Sheikh, R., Jacob, H.A.C., Gerber, C., 2000. Influence of version on loading pattern of the glenoid components: an experimental investigation. J. Shoulder Elbow Surg. 9, 549.

Orr, T.E., Carter, D.R., Schurman, D.J., 1988. Stress analyses of glenoid component designs. Clin. Orthop., 217-224.

Ramaniraka, N.A., Rakotomanana, L.R., Leyvraz, P.F., 2000. The fixation of the cemented femoral component. Effects of stem stiffness, cement thickness and roughness of the cement-bone surface. J. Bone Joint Surg. Br. 82, 297-303.

Rice, J.C., Cowin, S.C., Bowman, J.A., 1988. On the dependence of the elasticity and strength of cancellous bone on apparent density. J. Biomech. 21, 155-168.

Schaffler, M.B., Burr, D.B., 1988. Stiffness of compact bone: effects of porosity and density. J. Biomech. 21, 13-16.

Schmolz, W., Gordon, D.R., Shields, A.J., Kirkwood, D., Grigoris, P., 2000. The effect of stem geometry on stresses within the distal cement mantle in total hip replacement. Technol. Health Care 8, $67-73$.

Stone, K.D., Grabowski, J.J., Cofield, R.H., Morrey, B.F., An, K.N., 1999. Stress analyses of glenoid components in total shoulder arthroplasty. J. Shoulder Elbow Surg. 8, 151-158.

Topoleski, L.D., Ducheyne, P., Cuckler, J.M., 1990. A fractographic analysis of in vivo poly(methyl methacrylate) bone cement failure mechanisms. J. Biomed. Mater. Res. 24, 135-154.

Torchia, M.E., Cofield, R.H., Settergren, C.R., 1997. Total shoulder arthroplasty with the Neer prosthesis: long-term results. J. Shoulder Elbow Surg. 6, 495-505.

van der Helm, F.C., 1994. Analysis of the kinematic and dynamic behavior of the shoulder mechanism. J. Biomech. 27, 527550

Walch, G., Badet, R., Boulahia, A., Khoury, A., 1999. Morphologic study of the glenoid in primary glenohumeral osteoarthritis. J. Arthroplasty 14, 756-760.

Walker, P.S., 1997. Human joints and their artificial replacements, Springfield, IL, Thomas.

Wirth, M.A., Rockwood Jr., C.A., 1996. Complications of total shoulder-replacement arthroplasty. J. Bone Joint Surg. Am. 78, 603-616.

Yeh, O.C., Keaveny, T.M., 2001. Relative roles of microdamage and microfracture in the mechanical behavior of trabecular bone. J. Orthop. Res. 19, 1001-1007. 